DOI: https://doi.org/10.34069/RA/2021.8.02

Volumen 4, Número 8/julio-diciembre 2021

Peña Rodríguez, A., \& Lince Acosta, N.M. (2021). Papel de las teorías y los modelos científicos en la enseñanza-aprendizaje de la lectura en el nivel primario. Revista Científica Del Amazonas, 4(8), 19-28. https://doi.org/10.34069/RA/2021.8.02

\title{
Papel de las teorías y los modelos científicos en la enseñanza-aprendizaje de la lectura en el nivel primario
}

\section{Rol of the theories and the scientific models in the teaching-learning of the reading in the primary level}

Recibido: 18 de marzo de $2021 \quad$ Aceptado: 25 de mayo de 2021

\author{
Autores: \\ Armando Peña Rodríguez ${ }^{12}$ \\ Nora Maite Lince Acosta ${ }^{13}$
}

\section{Resumen}

Las ideas presentadas en el trabajo tienen como objetivo explicar la importancia de la teoría y los modelos científicos para dirigir el proceso de la enseñanza y el aprendizaje de la lectura en el nivel primario. Para satisfacer las expectativas planteadas, se aplicaron métodos del nivel teórico, tales como: el análisis-síntesis y la inducción- deducción, en el estudio de la literatura especializada. La metodología empleada consistió en consultar varias fuentes: impresas, digital y documentos de trabajo para la didáctica de la lengua en el nivel primario. Como resultados más importantes se pueden destacar: primero, el consenso de todos los especialistas al considerar que el aprendizaje de la lectura es muy difícil para los niños, por su naturaleza compleja y los procesos psicolingüísticos implicados, de ahí la variedad de teorías y modelos que proponen; segundo, la metodología de enseñanza-aprendizaje, aunque mantiene una secuencia estandarizada, actualmente posee otros puntos de vista que la actualizan con respecto al nivel alcanzado por la ciencia.

Palabras claves: teorías, modelos, lectura, enseñanza, aprendizaje.

\begin{abstract}
The ideas presented in the work have as objective to explain to the importance of the theory and the scientific models to lead the process of the teaching-learning of the reading in the primary level. To satisfy the outlined expectations, methods of the theoretical level were applied, such as: the analysis-synthesis and the induction - deduction, in the study of the specialized literature. The used methodology consisted on consulting several sources: printed, digital and work documents for the didactics of the language in the primary level. As more important results it can stand out: first, the consent of all the specialists when considering that the learning of the reading is very difficult for the children, for its complex nature and the psycholinguistic processes implied, hence the variety of theories and models that propose; second, the teaching-learning methodology, although it maintains a standardized sequence, at the moment it possesses other points of view that upgrade it with regard to the level reached by the science.
\end{abstract}

Keywords: theories, models, reading, teaching, learning.

${ }^{12}$ MSC, Prof asistente, Universidad de Oriente. CUM Palma Soriano. Cuba, https://orcid.org/0000-0002-8219-9433

${ }^{13}$ MSC, Prof. Auxiliar, Universidad de Oriente. CUM Palma Soriano, Cuba, https://orcid.org/0000-0001-9940-9567 


\section{AMAZONANAS}

\section{Introducción}

La ciencia ha alcanzado un desarrollo notable en la actualidad: se han elaborado nuevas tecnologías y han surgido nuevos conceptos en todas las ramas del saber humano, por tanto, ha cambiado el modo de ver el mundo en general. Gracias a las teorías y modelos científicos elaborados en el devenir histórico de la humanidad, el hombre puede disfrutar hoy de un modo de vida superior.

El papel de la teoría en la actividad práctica del hombre, así como la utilidad y limitaciones de los modelos científicos, es abordado ampliamente en la literatura especializada. Desde el punto de vista filosófico, los criterios son diversos, pero la concepción dialécticomaterialista del conocimiento es el que se considera más apropiado para el análisis del tema.

Es por eso que en la primera parte del trabajo aparece un tópico dedicado al análisis de las teorías y los modelos científicos en general con un enfoque dialéctico-materialista. A continuación, se presenta otra parte para explicar su relación con el proceso de enseñanzaaprendizaje de la lectura en el nivel primario, particularizando en las concepciones didácticometodológicas que lo sustentan.

Se plantea como objetivo fundamental, explicar la importancia de la teoría y los modelos científicos para dirigir el proceso de enseñanza-aprendizaje de la lectura en la Enseñanza Primaria.

\section{Metodología}

Para lograr el mismo, se aplicaron métodos del nivel teórico, tales como: el análisissíntesis y la inducción- deducción, en el estudio de la literatura especializada.

\section{Resultados y Discusión}

\section{¿Cuál es el logro más importante alcanzado por el hombre en el desarrollo histórico- social?}

Si se inicia la enumeración de ejemplos para responder la pregunta anterior, seguramente llevaría varias horas de meditación y muchas páginas escritas. Sin embargo, pudiera llegarse a la conclusión que el desarrollo científico-técnico es lo que distingue a la comunidad humana en lo que a logros se refiere. No existe esfera de la actividad cotidiana donde los aportes de la ciencia y la técnica no estén presentes, desde encender un bombillo, acontecimiento que deslumbró a José Martí y sus contemporáneos en el siglo XIX, hasta enviar señales electromagnéticas al espacio cósmico para indagar sobre la posible existencia de otras civilizaciones.

En el transcurso histórico de la humanidad hubo etapas en que era posible discernir con facilidad qué es técnica y qué es ciencia. Hoy las fronteras entre ambas son poco nítidas, por lo que resulta casi imposible separarlas. Sin embargo, para un análisis teórico del asunto, es posible dirigir las miradas hacia cada una de ellas por separado.

\section{¿Qué es la ciencia?}

En este trabajo el objetivo no es profundizar conceptualmente en el término ciencia y lo que ello implica, como sería entrar en consideraciones sobre la tecnología, tecnociencia, etc. Por tanto, estos conceptos son utilizados según su relación con el tema propuesto y a partir de las posiciones asumidas al definirlos, como es, por ejemplo: que los modelos y las teorías son elementos inseparables en el desarrollo de la ciencia. 
En el texto LA CIENCIA Y LA TECNOLOGIA COMO PROCESOS SOCIALES. Lo que la educación científica no debería olvidar, documento pdf, sin fecha, el profesor cubano Jorge Núñez Jover hace un análisis del concepto y concluye citando a Kröber, el cual plantea que:

...entendemos la ciencia no sólo como un sistema de conceptos, proposiciones, teorías, hipótesis, etc., sino también, simultáneamente, como una forma específica de la actividad social dirigida a la producción, distribución y aplicación de los conocimientos acerca de las leyes objetivas de la naturaleza y la sociedad. Aún más, la ciencia se nos presenta como una institución social, como un sistema de organizaciones científicas, cuya estructura y desarrollo se encuentran estrechamente vinculados con la economía, la política, los fenómenos culturales, con las necesidades y las posibilidades de la sociedad dada. (Kröber, 1986, citado por Núñez Jover, pág. 20).

Otro ejemplo de definición de ciencia, es la que ofrecen García y sus colaboradores varios autores en el texto Bases de la investigación educativa y sistematización de la práctica pedagógica: ellos dicen que es "... .un conjunto de conocimientos racionales, ciertos o probables, obtenidos metódicamente, sistematizados y verificables, que hacen referencia a objetos de una misma naturaleza." (García, Lanuez y otros, 2009, p. 124).

En ambos casos, para que haya ciencia deben existir conocimientos, los cuales alcanzan un alto nivel de abstracción, pues deben revelar lo oculto, lo que está más allá de nuestros sentidos, para explicarlo, es necesario un proceso de abstracción muy complejo, que arroja como resultado una teoría. La búsqueda de esos conocimientos implica una metódica, profesionales especialmente preparados en determinadas áreas de la cultura, pues la ciencia pertenece a la cultura.

En los últimos años se discute acerca del papel de la ciencia en la vida social. En Cuba constituye una política de primer orden poner la ciencia en función del desarrollo, sobre todo en aquellas áreas que ayudan a resolver los problemas que afectan directamente a la población.

\section{La teoría}

En la actualidad se ha difundido la idea de que el conocimiento tiene determinada estructura en el cerebro del hombre. Esta aseveración se sustenta en los resultados de investigaciones recientes en el campo de la psicología. Al respecto, resulta conveniente destacar, de forma resumida, los principales hallazgos de De Vega (1994) recogidos en su obra "Introducción a la Psicología cognitiva". Allí destaca tres maneras en que los individuos se representan mentalmente la información: imágenes, proposiciones y conceptos y categorías. Aunque las tres han recibido críticas, la generalidad de los especialistas actuales asume esa propuesta.

a) El conocimiento posee la estructura siguiente:

- Estructura de listas

- Redes semánticas

- Esquemas y prototipos (con carácter semántico)

b) Los formatos de las representaciones.

- Imágenes (analogía con la imagen perceptual)

- Verbal o lingüístico (palabra, frase, texto que se utiliza en el lenguaje)

- Proporcional (formalización de la información según la lógica de los predicados) 


\section{AMAZÉNAS}

c) Tipos de conocimientos representados en la memoria.

- Declarativos (conceptos). Tienen carácter consciente, son modificables y pueden ser recodificados en forma de imágenes verbales y no verbales.

- Procedimentales (acciones que no son comunicables)

Esas representaciones mentales se alcanzan como resultado del tránsito por los niveles del cocimiento: el concreto-sensible y el lógico-abstracto.

De acuerdo con lo expresado anteriormente, el desarrollo del conocimiento es fundamental para el origen y evolución de las teorías.

Por otra parte, los individuos aprenden, no solo con la interacción de las diferentes estructuras de la esfera cognitiva y sus funciones. Hay que tener en cuenta que el ser humano es el resultado de la interacción de tres factores: lo psíquico, lo bilógico y lo social, los cuales adquieren un dinamismo transformador en la actividad.

Precisamente, la actividad es la esencia mediante la cual se debe contextualizar el conocimiento, vista en su carácter teórico-práctica. De ella dependen el surgimiento, desarrollo, conservación y divulgación del conocimiento.

De acuerdo con este punto de vista, los distintos tipos de representaciones del conocimiento están caracterizados por el contexto, de manera que tiene una carga ideológica, sobre todo en aquellos que se derivan de las investigaciones en el área de las ciencias sociales. Al respecto, Rodríguez expresó que “...el saber filosófico representa un tipo de conocimiento que es simultáneamente informativo o de contenido, y valorativo." Como es obvio, se refiere al conocimiento filosófico propiamente dicho, sin embargo, toda teoría en el área de las ciencias sociales descansa en un soporte filosófico, por ser esta ciencia la más general de todas. Más adelante plantea que el conocimiento filosófico es diferente al científico-natural "...en virtud de que este último no lleva implícito en sus contenidos la valoración o el compromiso ideológico." (Rodríguez Uguido Z. 2017, p. 29)

Ser consecuente con estas ideas supone que formalmente el conocimiento posee una estructura en la psique, pero su contenido asume los rasgos de la ciencia particular en cuestión.

\section{¿Qué es la teoría y qué papel desempeña en la época actual?}

En el Diccionario de Filosofía se define la teoría como “....sistema de conocimientos fidedignos sistematizados que ofrece una representación íntegra de las regularidades y concatenaciones de la realidad y que describe, explica y predice el funcionamiento de un conjunto determinado de sus componentes". (Colectivo de autores, 1984, p.223).

Según este punto de vista, en la teoría los conocimientos no pueden presentarse aisladamente. Deben interrelacionarse formando una unidad dialéctica (carácter de sistema) deben ser el resultado de estudios conscientes de la realidad objetiva, desde diversos puntos de vista.

La teoría es el resultado de un largo proceso de desarrollo histórico-social del hombre. $\mathrm{Al}$ igual que el conocimiento, evoluciona en estrecha relación con las particularidades de las etapas del desarrollo social. Asimismo, está vinculada a las condiciones concretas que caracterizan los contextos sociales. 
La formación de teorías aisladas o relativamente aisladas y el movimiento hacia una teoría única, generalizada, son dos procesos concatenados como fases del conocimiento científico. Ellas pasan por estadíos sucesivos en los que se retoman los aspectos positivos de la anterior. Por otra parte, la acumulación gradual de teorías imprime nuevas cualidades a la teoría generalizadora, la que hace surgir nuevos problemas científicos y, como consecuencia, otras teorías.

Otra perspectiva en el análisis del papel que desempeña la teoría en la época actual, permite determinar tres dimensiones:

- Dimensión política.

- Dimensión social.

- Dimensión económica.

Dimensión política. La política es una forma de la conciencia. Esto quiere decir que tiene carácter clasista porque es función de ella perfeccionar los mecanismos que sustentan la posición de las clases sociales respecto a los medios de producción y los resultados del trabajo manual e intelectual. En este sentido, el estado, las instituciones y organismos creados con ese fin desempeñan el rol fundamental, cuya arma ideológica es más perfecta si las teorías que las sustentan son fuertes.

La teoría científica explica, describe y fundamenta la realidad objetiva tal como es, pero el rumbo que ellas toman está en dependencia de los objetivos clasistas con que se emplean. De ahí que adquieran un matiz ideológico, a veces claro a primera vista, pero otras veces sutiles.

Dimensión social. La teoría también desempeña un papel importante desde el punto de vista social. Muchas naciones que actualmente presentan características típicas del capitalismo, se diferencian entre sí por el nivel de desarrollo alcanzado. En los países más ricos el panorama social está menos depauperado que en los países capitalistas pobres, donde existen serios problemas de salud, educación, vivienda y otros males típicos del subdesarrollo.

Un ejemplo que ilustra lo dicho anteriormente es el uso que se la da al idioma español en Internet. Según publicaciones recientes, las informaciones que circulan en el mundo a través de la red de redes aparecen en idioma inglés en un porciento absolutamente mayor que en otros idiomas.

En otros países que pueden catalogarse de excepciones por el nivel de justicia social alcanzado, la teoría científica es una fuerza que impulsa acciones concretas para mejorar las condiciones de vida. En el orden social, son de gran utilidad las teorías que fundamentan cómo perfeccionar la educación de las nuevas generaciones, el desarrollo de la cultura general de todo el pueblo, la atención a personas discapacitadas física o mentalmente y cómo perfeccionar no solo la estructura, sino también las relaciones en la sociedad.

\section{Dimensión económica.}

El primer papel de la teoría en la dimensión mencionada, ha sido, y es, desentrañar la esencia de la actividad económica, anidada en las ciencias particulares, cuyos objetos, sistema categorial y métodos de investigación se corresponden con el proceso de producción y distribución de bienes materiales.

El examen por separado de las tres dimensiones señaladas y el papel de la teoría en cada caso, contempla el hecho de que ellas no se manifiestan aisladamente en la práctica, pues la 


\section{AMAZÉNAS}

estrecha relación entre la base económica y la superestructura de la sociedad, trae aparejado la unidad dialéctica entre todos los fenómenos económicos, políticos y sociales.

\section{Los modelos científicos. Utilidad y limitaciones.}

Los modelos científicos tienen carácter histórico-social. Igual que las teorías, ellos se enmarcan en los niveles de desarrollo alcanzado por la humanidad.

Existen modelos físicos, palpables. Indudablemente, esos modelos son de mucha importancia desde el punto de vista técnico. Pero en la actualidad el término modelo tiene otras connotaciones semánticas.

También se refiere a objetos de la realidad objetiva que no se perciben mediante los órganos sensoriales. Entonces el lenguaje, oral o escrito, se convierte en el medio más apropiado para representar los objetos no perceptibles para nuestros sentidos.

\section{¿Qué es un modelo científico?}

Es una representación del conocimiento humano sobre un objeto o fenómeno expresado verbalmente y que explica propiedades y relaciones de los elementos que integran aquella parte de la sociedad, el pensamiento o la naturaleza que resulta de interés para el hombre en la transformación del mundo para su beneficio.

Como parte de la representación descriptiva del objeto en su estado actual, el modelo científico debe reflejar con claridad en qué parte, relaciones o características se encuentra la esencia del problema, lo cual depende de la agudeza con que sea abordado por el investigador.

Pero el modelo científico no solo representa al objeto tal como es en un contexto dado, sino que puede ofrecer una imagen previa del estado futuro deseado (objeto ideal futuro). También desempeña el papel de objeto- sustituto, ya que es imposible, por ejemplo, llevar a una mesa de trabajo, a un forum, simposio u otros contextos de análisis, a procesos tan complejos como la enseñanza-aprendizaje de una materia. Allí, se lleva el modelo científico que lo representa, expresado en el sistema de teorías, proposiciones y conceptos propios de la ciencia en cuestión.

\section{¿Cuáles son las limitaciones más sobresalientes de los modelos científicos?}

Los modelos con proyección futura ofrecen una visión anticipada (reflejo ideal) del objeto. La calidad del tránsito de lo abstracto a lo concreto no está regulada por la voluntad de los hombres que "hacen ciencia", sino por aquellos que los aplican en la práctica, ya sea en la producción de bienes materiales, en la transformación de la naturaleza o en los procesos sociales. Por tal razón, los modelos dan lugar a interpretaciones variadas en la actividad práctica. Al exigir cierta preparación teórico-metodológica para su aplicación, están a merced de la praxis social y del propio desarrollo de la ciencia, quien pueden marcarle obsolescencia en pocos años.

De acuerdo con el desarrollo individual de cada hombre o la sociedad en su conjunto, la práctica enriquece los modelos proyectados, pero también puede negarlo total o parcialmente. Por otra parte, su generalización está determinada por las condiciones concretas de los contextos donde se desarrolla la actividad específica de los hombres.

\section{La teoría, los modelos científicos y el proceso de enseñanza-aprendizaje de la lectura.}

Las concepciones teóricas que abordan la lectura han evolucionado a través del tiempo. Algunas focalizan su naturaleza y otras su metodología. Actualmente se observa una franca lucha 
de teorías en los dos campos mencionados (naturaleza y metodología de la lectura). Este es uno de los elementos que permite dar argumento para demostrar el desarrollo científico alcanzado por las ciencias particulares que sirven de base a la enseñanza de la lectura.

Si hay un componente de la lengua priorizado en las investigaciones, ese es el caso de la lectura. Como ejemplos pueden presentarse los modelos creados a partir de los estudios con enfoques psicolingüísticos. Desde el punto de vista de la psicología cognitiva, predominan tres modelos que tratan de explicar la naturaleza de la lectura.

Según Manuel de Vega (De Vega, 1984), los modelos de lectura, vigentes todavía, comenzaron a difundirse en la década de los años setenta. En resumen, él refiere los siguientes:

- Modelo ascendente: Considera que la lectura se inicia con la consideración de las pistas gráficas, lexicales, sintácticas, contextuales y estructurales que aparecen en el texto y terminan con la construcción del significado en la mente del lector. Por tanto, se les da mayor importancia a los procesos perceptivos-visuales que tienen lugar durante la lectura.

- Modelo descendente: El proceso de leer es inverso al descrito anteriormente porque se inicia en la mente del lector y culmina en la página escrita.

- Modelo interactivo: La reconstrucción del significado del texto es el resultado de consideraciones interactivas de dos tipos de información: la que posee el lector y la que aporta el texto. Afirman que para comprender la lectura es necesario el empleo simultáneo de las dos.

Algunos especialistas opinan que el primero de estos modelos concibe la lectura como un proceso de "abajo-arriba" y el segundo de "arriba-abajo.

La modelación de la lectura como objeto arroja resultados evidentemente contradictorios. Cabe ahora retomar lo antes dicho respecto a la lucha de teorías.

¿En cuál de ellos se refleja el proceso de lectura de forma correcta?

¿La práctica corrobora lo expresado en cada uno?

¿Hasta qué punto son útiles para el maestro de la escuela primaria?

La primera pregunta promueve otras interrogantes. Si se parte del supuesto psicológico que cada individuo posee características particulares en cuanto al desarrollo de su personalidad (afectivo-cognitivo), entonces:

¿El proceso de lectura es igual en todos los lectores?

¿Cada lector postula un modelo diferente no representado aún por los investigadores? o ¿Habrá que aceptar la idea de clasificar los lectores según los patrones establecidos por cada modelo?

Hasta el momento ellas exigen continuar profundizando en la naturaleza de la lectura, porque existen otros indicadores no cuantificados suficientemente, al menos en los textos consultados, que permitan precisar teorías, como es el caso de las etapas del aprendizaje de la lectura. Por ejemplo: al concebir el modelo ascendente es más fácil justificarlo mediante la observación de individuos en la etapa de adquisición del código de la lengua, para la que muchas veces los métodos sintéticos (tránsito del grafema-fonema a la idea) son los más empleados para dirigir el proceso de enseñanza-aprendizaje de la lectura, momento en que no se aprecian las características del modelo descendente, según el cual los niños de 1. grado deberán "elaborar hipótesis" y después corroborarlas durante la lectura de sílabas, palabras, frases y oraciones, cuyos 


\section{AMAZONANAS}

significados deben relacionar con sus conocimientos previos, porque no dominan la mecánica de la lectura.

Por tanto, la competencia alcanzada para la lectura es un factor que determina el "ascenso" o "descenso" cuando se lee un texto escrito. Lo mismo ocurre con el contenido del texto y el dominio que posee el lector del mismo. Este es un elemento discutible porque, según lo ya expresado en páginas anteriores, el conocimiento se elabora por la interacción de varios factores, entre los que se encuentra el contexto.

Al hablar del contexto, se incluyen todos los factores que tributan al desarrollo humano, tanto lo interno como lo externo. Este último contiene a la cultura, la cual proporciona espacios para el aprendizaje en correspondencia con la etapa o momento del desarrollo del individuo. Así, el conocimiento previo, la cultura individual, es consecuencia del contexto, por tanto, diferente por su contenido y la interrelación que pueda establecer el individuo en cada situación. Esto quiere decir que, al leer, el individuo puede o no puede dominar de antemano el contenido de un texto.

Se está de acuerdo en que todo lector posee un conocimiento previo, de diversos tipos, el cual le permite predecir de qué trata un texto leyendo solamente el título, un enunciado, fragmento, etc. pero, ¿siempre ocurre así?

Según los puntos de vistas del modelo descendente, se pudiera plantear que, al partir de una situación comunicativa en la que se realiza un trabajo oral previo para iniciar el aprendizaje de un nuevo fonema, sílabas, palabras y oraciones, se está partiendo de la idea para corroborarla en la lectura de las unidades lingüísticas mencionadas, pero en este caso, es una idea prediseñada por un factor externo al sistema cognitivo de los alumnos: el docente. Responde a requerimientos puramente metodológicos y no a intenciones personales del lector, a objetivos, motivos y necesidades de comunicación, disfrute o goce estético, que son los móviles de la lectura.

Es arriesgado afirmar de forma absoluta que la lectura es un proceso descendente para verificar hipótesis, pues está en dependencia del desarrollo alcanzado por el individuo.

La lectura, como actividad individual, se adecua a objetivos diferentes: para la solución de tareas durante el estudio, la recreación, localizar informaciones, ampliar un conocimiento, etc. Ellos predisponen al lector para procesar la información de un texto escrito

\section{Metodología de la lectura}

Se considera una necesidad inmediata partir del presupuesto general en que se basa la enseñanza-aprendizaje de la lectura. Según el sistema educativo vigente, las didácticas especiales, aquellas que sostienen el qué y el cómo se enseña, están alineadas con los presupuestos psicopedagógicos generales basados en una política de estado. Por tanto, aún cuando el docente asume estrategias diferenciadas creadas personalmente, su proyección metodológica responde al enfoque general que le plantea el sistema educativo.

En Cuba, el sistema educativo está basado en un modelo que postula el desarrollo integral de la personalidad, cuyos fundamentos tiene una base en las ideas martianas, marxista leninista y fidelista. De ahí que los fundamentos psicopedagógicos en que se sustenta, tienen en cuenta las ideas salidas de estas fuentes de pensamiento. Al mismo tiempo, al considerar al individuo como un ser en desarrollo, se asumen los criterios que mejor explican el proceso de formación de la personalidad. Tal es el caso de las ideas de Vigostky, de mucha influencia en la educación.

La visión de que el aprendizaje puede conducir al desarrollo es muy influyente en el sistema educativo de muchos países. Esta concepción ayuda a los docentes para diseñar 
condiciones que propician el desarrollo integral de los alumnos, tales como: estrategias, juegos, metodologías y tareas donde se integran la educación y la instrucción.

En la actualidad, el enfoque integrador en la didáctica de la lengua ubica a la lectura como uno de sus componentes funcionales más importantes. Es así como ella se convierte en el eje central que sustentan casi todas las tareas docentes, no solo en la didáctica de la lengua, sino de todas las materias. Su esencia consiste en dirigir el proceso de enseñanza-aprendizaje de la lectura integrado al desarrollo de conocimientos y habilidades para el uso efectivo de la lengua materna. Se deben atender tres componentes funcionales: la lectura y comprensión de textos, lectura y análisis de textos y la construcción de textos.

Para Garriga (2002), estos componentes se aíslan para su estudio, pero en la práctica el docente debe concebirlos como un sistema, para lo cual propone actividades que ejemplifican cómo respetar su carácter sistémico. Las sugerencias que propone se ajustan al nivel medio (secundaria básica y preuniversitario) pero modificando las actividades en cuanto a la forma y el contenido, o creando otras, el docente de primaria puede llevar a sus aulas las ideas del enfoque integral a un grado específico, de hecho, hoy es una norma en la didáctica de la Lengua Española como asignatura.

\section{Conclusiones}

Después de haber aplicado los métodos seleccionados para el desarrollo del tema, se ha llegado a las conclusiones siguientes:

La teoría y los modelos científicos son de gran utilidad para la práctica, tanto en los procesos de investigación como en la producción de bienes materiales, la docencia, el deporte, la cultura, etc. Son el motor impulsor del desarrollo de la ciencia.

Las teorías y los modelos que se refieren a la lectura se han desarrollado en los últimos años y propician el constante perfeccionamiento del proceso de enseñanza-aprendizaje de la lectura.

Actualmente, el enfoque integrador es una posición teórica que da una visión más ajustada a los requerimientos de la lengua española, por las características de este idioma y por las oportunidades metodológicas que ofrece.

\section{Bibliografía}

Abrahan, W. (1981). Diccionario de terminología lingüística actual. Madrid: Editorial Gredos.

Álvarez Álvarez, L. (1996). La lectura: ¿pasividad o dinamismo? En Educación (Cuba). No. 89. Arias Leiva, G. (2000). Leer antes de leer. En Educación (Cuba), No. 101.

Aróstegui, J. M. (1978). Metodología del conocimiento científico. Ciudad de la Habana: Editorial de Ciencias Sociales.

Byron, J. (1994). La linguística del texto y el proceso de lectura. En Investigaciones para el desarrollo (Santo Domingo). Año 1, No 1, octubre.

Cabrera Delgado, L. (1992). La lectura como necesidad. La lectura como objeto real de esa necesidad. En junio como en enero (Cuba). No 10. Junio.

Colectivo de autores. (1986). Manual de Fisiología Marxista-leninista. La Habana: Editorial pueblo y Educación.

De Vega, M. (1994). Introducción a la Psicología Cognitiva. Madrid: Alianza Editorial.

Diccionario de filosofía. (1984). Moscú: Editorial Progreso.

Falcón de Ovalles, J. (1995) El conocimiento previo y el procesamiento del texto escrito. Estrategia para la utilización de este conocimiento. En Laurus (México), No. 1. 


\section{AMAZÓNAS}

García Batista, G. y otros. (2009). Fundamentos de la investigación educativa. Ciudad de La Habana: Editorial Pueblo y Educación.

García Batista, G., Lanuez Bayolo M. y et. al. (2009). Bases de la investigación educativa y sistematización de la práctica pedagógica. En García Batista, G. Fundamentos de la investigación educativa (p. 124). La Habana: Editorial Pueblo y Educación.

Garcia Pers, D. (1995). La enseñanza de la lengua materna en la escuela primaria (I parte). Ciudad de la Habana: Editorial Pueblo y Educación.

Garriga Valiente E. A. (2002). El tratamiento de los componentes funcionales: comprensión, análisis y construcción de textos. En Roméu Escobar A. Acerca de la enseñanza del español y la literatura. La Habana: Editorial Pueblo y Educación.

Morenza Padilla, L. Memoria semántica. (1993). Estructura de conocimiento y aprendizaje de la lectura. En Educación (Cuba). No 84, enero-abril.

Morenza Padilla, L.y Orlando Torres Camacho. (1998). Escuela histórico-cultural. Liliana Morenza Padilla. En Educación (Cuba). No 93, enero-abril.

Peña Rodríguez, A. Y Lince Acosta N. M. (2018) Qué es leer y qué fuentes de información pueden ser leídas. Revista Atlante: cuadernos de Educación y Desarrollo, II etapa, octubre 2018, ISSN: 1989-4155.

Plá, León R. L. (2017). La teoría del conocimiento del marxismo. En Filosofía Marxista I. La Habana: Editorial Universitaria Félix Varela.

Rodríguez Uguido, Z. (2017). El pensamiento teórico como objeto de la ciencia filosófica marxista-leninista. En Suárez Martín, L. et. al. Filosofía Marxista I. La Habana: Editorial Félix Varela.

Romeu Escobar, A. (1994) Comunicación y enseñanza de la lengua. En Educación (Cuba), No. 83 .

Scot Gray, W. (1958). La enseñanza de la lectura y la escritura [s.p.i.]. 